\title{
Plane curves as Pfaffians
}

\author{
ANITA BUCKLEY AND TOMAŽ KOŠIR
}

\begin{abstract}
Let $C$ be a smooth curve in $\mathbb{P}^{2}$ given by an equation $F=0$ of degree $d$. In this paper we parametrise all linear Pfaffian representations of $F$ by an open subset in the moduli space $M_{C}\left(2, K_{C}\right)$. We construct an explicit correspondence between Pfaffian representations of $C$ and rank 2 vector bundles on $C$ with canonical determinant and no sections.
\end{abstract}

Mathematics Subject Classification (2010): 14H60 (primary); 14D20, 15A15, 15A54 (secondary).

\section{Introduction}

Let $k$ be an algebraically closed field and $C$ an irreducible curve in $\mathbb{P}^{2}$ defined by a polynomial $F\left(x_{0}, x_{1}, x_{2}\right)$ of degree $d$. We consider the following question. For given $C$ (and $F$ ) find a $2 d \times 2 d$ skew-symmetric matrix

$$
A=\left[\begin{array}{ccccc}
0 & L_{12} & L_{13} & \cdots & L_{12 d} \\
-L_{12} & 0 & L_{23} & \cdots & L_{22 d} \\
-L_{13} & -L_{23} & 0 & & \\
\vdots & \vdots & \ddots & \vdots \\
-L_{12 d} & -L_{22 d} & \cdots & 0
\end{array}\right]
$$

with linear forms $L_{i j}=a_{i j}^{0} x_{0}+a_{i j}^{1} x_{1}+a_{i j}^{2} x_{2}$ such that

$$
\operatorname{Pf} A\left(x_{0}, x_{1}, x_{2}\right)=c F\left(x_{0}, x_{1}, x_{2}\right) \text { for some } c \in k, c \neq 0 \text {. }
$$

Such a matrix $A$ is called a linear Pfaffian representation of $C$. Its cokernel is a rank 2 vector bundle on $C$. Throughout the paper we identify vector bundles with locally free sheaves.

Two Pfaffian representations $A$ and $A^{\prime}$ are equivalent if there exists $X \in \mathrm{GL}_{2 d}(k)$ such that

$$
A^{\prime}=X A X^{t}
$$

Received June 3, 2009; accepted in revised form April 8, 2010. 
A locally free sheaf $\mathcal{E}$ of rank 2 is stable if for every invertible sheaf $\mathcal{E} \rightarrow \mathcal{F} \rightarrow 0$ holds

$$
\operatorname{deg} \mathcal{F}>\frac{1}{2} \operatorname{deg} \mathcal{E}
$$

It is semistable if $>$ is replaced by $\geq$.

We find all linear Pfaffian representations of $C$ (up to equivalence) and relate them to the moduli space $M_{C}\left(2, K_{C}\right)$ of semistable rank 2 vector bundles on $C$ with canonical determinant. An explicit construction of representations from the global sections of rank 2 vector bundles with canonical determinant and no sections is given.

In general the elements of $A$ can be homogenous polynomials of various degrees. Such Pfaffian representations are considered in Theorem 3.6. A good survey of the linear algebra of Pfaffians can be found in [8, Appendix D]. In [9, Chapter V. 2] Hartshorne identifies the theory of rank 2 sheaves with the theory of ruled surfaces over $C$.

Study of Pfaffian representations is strongly related to and motivated by determinantal representations. A linear determinantal representation of $C$ is a $d \times d$ matrix of linear forms

$$
M=x_{0} M_{0}+x_{1} M_{1}+x_{2} M_{2}
$$

satisfying

$$
\operatorname{det} M=c F,
$$

where $M_{0}, M_{1}, M_{2} \in \operatorname{Mat}_{d}(k)$ and $c \in k, c \neq 0$. Here $\operatorname{Mat}_{d}(k)$ is the algebra of all $d \times d$ matrices over $k$. Two determinantal representations $M$ and $M^{\prime}$ are equivalent if there exist $X, Y \in \mathrm{GL}_{d}(k)$ such that

$$
M^{\prime}=X M Y .
$$

There are many more Pfaffian than determinantal representations. Indeed, every determinantal representation $M$ induces decomposable Pfaffian representation

$$
\left[\begin{array}{cc}
0 & M \\
-M^{t} & 0
\end{array}\right]
$$

Note that the equivalence relation is well defined since

$$
\left[\begin{array}{cc}
0 & X M Y \\
-(X M Y)^{t} & 0
\end{array}\right]=\left[\begin{array}{cc}
X & 0 \\
0 & Y^{t}
\end{array}\right]\left[\begin{array}{cc}
0 & M \\
-M^{t} & 0
\end{array}\right]\left[\begin{array}{cc}
X^{t} & 0 \\
0 & Y
\end{array}\right] .
$$

Nonequivalent linear determinantal representations are in bijection with line bundles on $C$ and they can be parametrised by the non exceptional points on the Jacobian variety of $C$. To any compact Riemann surface $X$ one can associate the pair $(J X, \Theta)$, the Jacobian and the Riemann theta function. The geometry of the pair is strongly related to the geometry of $X$. This gives an idea that higher rank vector 
bundles define a non-abelian analogue of the Jacobian called moduli space firstly due to the mathematicians of the Tata Institute [15]. Much later physicists in Conformal Field Theory introduced pairs of moduli spaces and determinant line bundles on these moduli spaces [20]. This has made a clear analogy with the Jacobian pair.

A brief outline of the paper is the following. In Section 2 we recall the parametrisation of linear determinantal representations by points on the Jacobian variety due to Vinnikov [22]. We use similar ideas in Sections 3 and 4 to parametrise all linear Pfaffian representations by points in an open subset of the moduli space $M_{C}\left(2, K_{C}\right)$. In the third section we give an explicit construction of the correspondence between Pfaffian representations and vector bundles with certain properties. In the fourth section we relate these vector bundles to the points on $M_{C}\left(2, K_{C}\right)$ not on the subvariety cut out by Cartier divisor $\Theta_{2, K_{C}}$. In Section 5 we consider decomposable bundles which are parametrised by an open set on the Kummer variety. Pfaffian representation arising from a decomposable bundle $\mathcal{F} \oplus$ $\left(\mathcal{F}^{-1} \otimes \mathcal{O}_{C}(d-1)\right)$ is exactly the decomposable representation

$$
\left[\begin{array}{cc}
0 & M \\
-M^{t} & 0
\end{array}\right]
$$

where $M$ is the determinantal representation corresponding to $\mathcal{F} \cong$ Coker $M$. We show in Section 6 that elliptic curves only allow decomposable Pfaffian representations. This is a special case of the general result that elliptic curves allow only decomposable vector bundles [3]. In the last section we compute Pfaffian representations of a genus 3 curve. To our knowledge there are not many results explicitly describing $M_{C}\left(2, K_{C}\right)$ for curves of higher genus. Methods considered in this paper could be applied to finding all Pfaffian representations and consequently the moduli space of genus $g>3$ curves.

Let us also mention that explicit construction of elementary transformations of Pfaffian representations of smooth plane curves is given in [4].

ACKNOWLEDGEMEnTs. The authors would like to thank Emilia Mezzetti for suggesting to study Pfaffian representations.

\section{Determinantal representations and the Jacobian}

There is a one to one correspondence between linear determinantal representations (up to equivalence) of $C$ and line bundles (up to isomorphism) on $C$ with certain properties. This well known result is summed up in the following theorem of Beauville [2, Proposition 3.1].

Theorem 2.1. Let $C$ be a plane curve defined by a polynomial $F$ of degree $d$ and let $L$ be a line bundle of degree $\frac{1}{2} d(d-1)$ on $C$ with $H^{0}(C, L(-1))=0$. Then 
there exists a $d \times d$ linear matrix $M$ with $\operatorname{det} M=F$ and an exact sequence

$$
0 \rightarrow \bigoplus_{i=1}^{d} \mathcal{O}_{\mathbb{P}^{2}}(-1) \stackrel{M}{\longrightarrow} \bigoplus_{i=1}^{d} \mathcal{O}_{\mathbb{P}^{2}} \rightarrow L \rightarrow 0 .
$$

Conversely, let $M$ be a linear $d \times d$ matrix with $\operatorname{det} M=F$. Then its cokernel is a line bundle of degree $\frac{1}{2} d(d-1)$ and $H^{0}(C$, Coker $M(-1))=0$.

Dolgachev explicitly described the above correspondence in [6].Vinnikov also gave an explicit construction of the correspondence in [22, Theorems 2-4]. Additionally he related determinantal representations to points on the Jacobian variety in the following way.

It is known [5, Theorem 1.1] that representations $M$ and $M^{\prime}$ are equivalent if and only if Coker $M$ and Coker $M^{\prime}$ are isomorphic sheaves. Therefore the problem of classifying all linear representations of $F$ (up to equivalence) it the same to finding all line bundles $\mathcal{L}$ with the property $\operatorname{deg} \mathcal{L}=\frac{1}{2} d(d-1)$ and $H^{0}(C, \mathcal{L}(-1))=0$. In order to simplify the notation we tensor the above by $\mathcal{O}(1)$ and consider line bundles with $\operatorname{deg} \mathcal{L}=\frac{1}{2} d(d-1)-d=\frac{1}{2} d(d-3)$ and $H^{0}(C, \mathcal{L})=0$. We call the bundles with this property non exceptional line bundles. Analogously, line bundles of degree $\frac{1}{2} d(d-3)$ and $H^{0}(C, \mathcal{L}) \neq 0$ are called exceptional. Recall a fundamental result in the theory of curves [1]:

Lemma 2.2. Let $C$ be of genus $g$. The exceptional divisor classes define a $g-1$ dimensional subvariety $W_{g-1}$ on the Jacobian variety $J$.

This proves the following theorem [22, Theorems 2-4]:

Theorem 2.3. All linear determinantal representations of $F$ (up to equivalence) can be parametrised by points on the Jacobian variety of $C$ not on the exceptional subvariety $W_{g-1}$.

\section{Classification of Pfaffian representations from the scratch}

In this section we consider the following problem. For a given homogeneous polynomial $F\left(x_{0}, x_{1}, x_{2}\right)$ of degree $d$ find all linear Pfaffian representations. The main result of this section is an elementary proof of Theorem 3.5 and explicit construction of representations from suitable vector bundles given in the proof of Proposition 3.4.

Following the ideas of Dolgachev [6] we formulate the problem geometrically and coordinate free. Let $U$ be a $2 d$ dimensional vector space. It is well known that $\bigwedge^{2} U$ can be identified with $2 d \times 2 d$ skew-symmetric matrices. Let $\Omega_{k}$ denote the set of elements $\sum_{i=1}^{k} v_{i} \wedge w_{i}$ in $\wedge^{2} U$ where $\operatorname{dim}\left\{v_{1}, \ldots, v_{k}, w_{1}, \ldots, w_{k}\right\}=2 k$. Elements of $\Omega_{k}$ are said to have irreducible length $k$ since they can be written as a sum of $k$ and not less than $k$ decomposable tensors in $\bigwedge^{2} U$. In [10] it is shown that 
Lemma 3.1. $\Omega_{k}$ can be identified with the set of all rank $2 k$ skew-symmetric matrices.

Proof. Specifically, let $e_{1}, \ldots, e_{2 d}$ be a basis for $U$ and $\left\{E_{i j}\right\}$ the standard basis for $2 d \times 2 d$ matrices. Then the bijection given by

$$
e_{i} \wedge e_{j} \mapsto E_{i j}-E_{j i},
$$

extends linearly to

$$
\sum_{i=1}^{2 d} \alpha_{i} e_{i} \wedge \sum_{j=1}^{2 d} \beta_{j} e_{j} \mapsto \sum_{i, j=1}^{2 d}\left(\alpha_{i} \beta_{j}-\alpha_{j} \beta_{i}\right)\left(E_{i j}-E_{j i}\right) .
$$

Next let $E$ be a 3 dimensional vector space and $\Phi$ a linear embedding

$$
\Phi: \mathbb{P}(E) \longrightarrow \mathbb{P}\left(\bigwedge^{2} U\right)
$$

Note that $\Phi$ corresponds to a skew-symmetric matrix with linear forms as its elements. Alternatively, $\Phi$ is an element in $E^{*} \otimes\left(\bigwedge^{2} U\right)$.

Let $\mathcal{P}_{d} \subset \mathbb{P}\left(\bigwedge^{2} U\right)$ be the hypersurface parametrising non-invertible skewsymmetric matrices. Choose a basis of $U$, then $\mathcal{P}_{d}$ is given by the Pfaffian of a skew-symmetric matrix. The inverse image of $\mathcal{P}_{d}$ under $\Phi$ is a plane curve of degree $d$ in $\mathbb{P}(E)$.

Let $C$ be a smooth plane curve defined by $F$. Assume that $C$ admits a Pfaffian representation $A$.

Lemma 3.2. For any $x \in C$ the corank of $A(x)$ equals 2.

Proof. Assume

$$
A=\left[a_{i j}^{0} x_{0}+a_{i j}^{1} x_{1}+a_{i j}^{2} x_{2}\right] \text { and } \operatorname{Pf} A=c F, c \neq 0 .
$$

Denote by $\mathrm{P}^{i j} A$ the Pfaffian of the $(2 d-2) \times(2 d-2)$ skew-symmetric matrix obtained by removing the $i$ th and $j$ th rows and columns from $A$. Then

$$
\frac{\partial F}{\partial x_{k}}(x)=\frac{1}{c} \sum_{i, j} a_{i j}^{k} \operatorname{Pf}^{i j} A(x) .
$$

If for some $x \in C$ all $2 d-2$ Pfaffian minors vanish, then $x$ must be a singular point of $F$. By our assumption $F$ is smooth, thus rank $A(x) \geq 2 d-2$ for all $x \in C$. This ends the proof because rank of skew-symmetric matrices is even and $\operatorname{det} A(x)=F^{2}(x)=0$. 
Define the Pfaffian adjoint of $A$ to be the skew-symmetric matrix

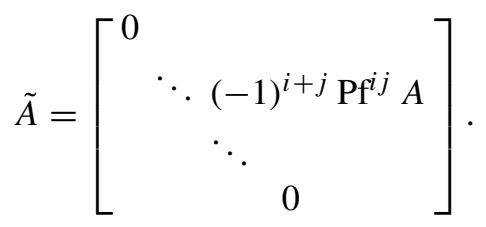

By analogy with determinants the following holds

$$
\tilde{A} \cdot A=\operatorname{Pf} A \cdot \operatorname{Id}_{2 d} .
$$

By Lemma 3.2 the cokernel of $A$ defines a rank 2 vector bundle over $C$. Since Pf $A=c F$ the cokernel can be obtained from $\tilde{A}$ by using identity (3.2). Indeed, for any point $x \in C$ every column of $\tilde{A}(x)$ is in Coker $A(x)$. The properties of Coker $A$ are described in the following proposition.

Proposition 3.3. Let $A$ be a Pfaffian representation of a smooth plane curve $C$ defined by a homogeneous polynomial $F$ of degree $d$. Then $\mathcal{E}=\operatorname{Coker} A$ is a rank 2 vector bundle on $C$ and

(i) $h^{0}(C, \mathcal{E})=2 d$,

(ii) $H^{0}(C, \mathcal{E}(-1))=H^{1}(C, \mathcal{E}(-1))=0$,

(iii) $\operatorname{det} \mathcal{E}=\bigwedge^{2} \mathcal{E}=\mathcal{O}_{C}(d-1)$.

Proof. By definition of $\mathcal{E}$ we have the exact sequence

$$
0 \rightarrow \bigoplus_{i=1}^{2 d} \mathcal{O}_{\mathbb{P}^{2}}(-1) \stackrel{A}{\longrightarrow} \bigoplus_{i=1}^{2 d} \mathcal{O}_{\mathbb{P}^{2}} \rightarrow \mathcal{E} \rightarrow 0
$$

Applying the functor $H^{i}\left(\mathbb{P}^{2}, *\right)$ to $(3.3)$ gives

$H^{0}\left(\mathbb{P}^{2}, \mathcal{O}_{\mathbb{P}^{2}}(-1)^{2 d}\right) \rightarrow H^{0}\left(\mathbb{P}^{2}, \mathcal{O}_{\mathbb{P}^{2}}^{2 d}\right) \rightarrow H^{0}\left(\mathbb{P}^{2}, \mathcal{E}\right) \rightarrow H^{1}\left(\mathbb{P}^{2}, \mathcal{O}_{\mathbb{P}^{2}}(-1)^{2 d}\right) \rightarrow \cdots$

0

$2 d$

where the bottom row denotes dimensions of the cohomology of projective space which is computed in [9, Theorem III.5.1]. Thus $\operatorname{dim} H^{0}\left(\mathbb{P}^{2}, \mathcal{E}\right)=2 d$. 
Next tensor (3.3) by $\mathcal{O}_{\mathbb{P}^{2}}(-1)$ and again apply the functor $H^{i}\left(\mathbb{P}^{2}, *\right)$. This gives a long exact sequence

$$
\begin{array}{cc}
H^{0}\left(\mathbb{P}^{2}, \mathcal{O}_{\mathbb{P}^{2}}(-2)^{2 d}\right) & \rightarrow H^{0}\left(\mathbb{P}^{2}, \mathcal{O}_{\mathbb{P}^{2}}(-1)^{2 d}\right) \rightarrow H^{0}\left(\mathbb{P}^{2}, \mathcal{E}(-1)\right) \rightarrow \\
0 & 0 \\
H^{1}\left(\mathbb{P}^{2}, \mathcal{O}_{\mathbb{P}^{2}}(-2)^{2 d}\right) & \rightarrow H^{1}\left(\mathbb{P}^{2}, \mathcal{O}_{\mathbb{P}^{2}}(-1)^{2 d}\right) \rightarrow H^{1}\left(\mathbb{P}^{2}, \mathcal{E}(-1)\right) \rightarrow \\
\| & \| \\
0 & 0 \\
H^{2}\left(\mathbb{P}^{2}, \mathcal{O}_{\mathbb{P}^{2}}(-2)^{2 d}\right) \rightarrow & \cdots \\
\| &
\end{array}
$$

where $H^{2}\left(\mathbb{P}^{2}, \mathcal{O}_{\mathbb{P}^{2}}(-2)^{2 d}\right) \cong H^{0}\left(\mathbb{P}^{2}, \mathcal{O}_{\mathbb{P}^{2}}(-1)^{2 d}\right)=0$ by Serre duality. Hence, we obtain that $H^{0}\left(\mathbb{P}^{2}, \mathcal{E}(-1)\right)=H^{1}\left(\mathbb{P}^{2}, \mathcal{E}(-1)\right)=0$.

Since $\mathcal{E}$ is supported on $C$ we proved (i) and (ii).

In order to prove (iii) apply the functor $\mathcal{H o m}_{\mathcal{O}_{\mathbb{P}^{2}}}\left(*, \mathcal{O}_{\mathbb{P}^{2}}(-1)\right)$ to (3.3). We get

$$
\begin{aligned}
0 & \rightarrow \mathcal{H} \operatorname{Hom}\left(\mathcal{E}, \mathcal{O}_{\mathbb{P}^{2}}(-1)\right) \rightarrow \mathcal{H} \operatorname{om}\left(\mathcal{O}_{\mathbb{P}^{2}}^{2 d}, \mathcal{O}_{\mathbb{P}^{2}}(-1)\right) \rightarrow \mathcal{H o m}\left(\mathcal{O}_{\mathbb{P}^{2}}(-1)^{2 d}, \mathcal{O}_{\mathbb{P}^{2}}(-1)\right) \rightarrow \\
& \rightarrow \mathcal{E} x t^{1}\left(\mathcal{E}, \mathcal{O}_{\mathbb{P}^{2}}(-1)\right) \rightarrow \mathcal{E} x t^{1}\left(\mathcal{O}_{\mathbb{P}^{2}}^{2 d}, \mathcal{O}_{\mathbb{P}^{2}}(-1)\right) \rightarrow
\end{aligned}
$$

where

$$
\begin{gathered}
\mathcal{H o m}\left(\mathcal{O}_{\mathbb{P}^{2}}^{2 d}, \mathcal{O}_{\mathbb{P}^{2}}(-1)\right) \cong\left(\mathcal{O}_{\mathbb{P}^{2}}^{2 d}\right)^{\vee} \otimes \mathcal{O}_{\mathbb{P}^{2}}(-1) \cong \mathcal{O}_{\mathbb{P}^{2}}(-1)^{2 d} \\
\mathcal{H} \operatorname{om}\left(\mathcal{O}_{\mathbb{P}^{2}}(-1)^{2 d}, \mathcal{O}_{\mathbb{P}^{2}}(-1)\right) \cong\left(\mathcal{O}_{\mathbb{P}^{2}}(-1)^{2 d}\right)^{\vee} \otimes \mathcal{O}_{\mathbb{P}^{2}}(-1) \cong \mathcal{O}_{\mathbb{P}^{2}}^{2 d}
\end{gathered}
$$

and $\mathcal{E} x t^{1}\left(\mathcal{O}_{\mathbb{P}^{2}}^{2 d}, \mathcal{O}_{\mathbb{P}^{2}}(-1)\right)=0$ by [9, Proposition III.6.3].

This implies that $\mathcal{E} x t^{i}\left(\mathcal{E}, \mathcal{O}_{\mathbb{P}^{2}}(-1)\right)=0$ for $i \neq 1$ and the above sequence is isomorphic to

$$
0 \rightarrow \bigoplus_{i=1}^{2 d} \mathcal{O}_{\mathbb{P}^{2}}(-1) \stackrel{A^{t}}{\longrightarrow} \bigoplus_{i=1}^{2 d} \mathcal{O}_{\mathbb{P}^{2}} \rightarrow \mathcal{E} x t^{1}\left(\mathcal{E}, \mathcal{O}_{\mathbb{P}^{2}}(-1)\right) \rightarrow 0
$$

Thus we obtain

$$
\begin{aligned}
& \mathcal{E} \cong \operatorname{Coker} A \cong \operatorname{Coker} A^{t} \cong \mathcal{E} x t^{1}\left(\mathcal{E}, \mathcal{O}_{\mathbb{P}^{2}}(-1)\right) \\
& \cong \mathcal{E} x t^{1}\left(\mathcal{E}, \mathcal{O}_{\mathbb{P}^{2}}(-3) \otimes \mathcal{O}_{\mathbb{P}^{2}}(2)\right) \mathcal{E} x t^{1}\left(\mathcal{E}, \mathcal{O}_{\mathbb{P}^{2}}(-3)\right) \otimes \mathcal{O}_{\mathbb{P}^{2}}(2) \\
& \cong \mathcal{H o m}_{C}\left(\mathcal{E}, \mathcal{O}_{C}(d-3)\right) \otimes \mathcal{O}_{\mathbb{P}^{2}}(2) \cong \mathcal{H o m}_{C}\left(\mathcal{E}, \mathcal{O}_{C}(d-1)\right) \\
& \cong \mathcal{E}^{\vee} \otimes \mathcal{O}_{C}(d-1)
\end{aligned}
$$


since by Serre duality

$$
\mathcal{E} x t^{1}\left(\mathcal{E}, \mathcal{O}_{\mathbb{P}^{2}}(-3)\right) \cong \mathcal{E} x t^{1}\left(\mathcal{E}, \omega_{\mathbb{P}^{2}}\right) \cong \mathcal{H o m}_{C}\left(\mathcal{E}, \omega_{C}\right) \cong \mathcal{H o m}_{C}\left(\mathcal{E}, \mathcal{O}_{C}(d-3)\right)
$$

Finally,

$$
\wedge^{2} \mathcal{E} \cong \mathcal{E} \wedge\left(\mathcal{E}^{\vee} \otimes \mathcal{O}_{C}(d-1)\right) \cong \mathcal{O}_{C}(d-1)
$$

In the sequel the reverse problem will be considered. We will give an explicit construction of Pfaffian representation from a vector bundle with properties (i)-(iii).

Proposition 3.4. Let $C$ be a smooth plane curve of degree $d$. To every rank 2 vector bundle $\mathcal{E}$ on $C$ with properties

(i) $h^{0}(C, \mathcal{E})=2 d$,

(ii) $H^{0}(C, \mathcal{E}(-1))=0$,

(iii) $\operatorname{det} \mathcal{E}=\bigwedge^{2} \mathcal{E}=\mathcal{O}_{C}(d-1)$

we can assign a Pfaffian representation $A_{\mathcal{E}}$. In particular, isomorphic bundles induce equivalent representations.

Proof. Let $U=H^{0}(C, \mathcal{E})$ be the $2 d$ dimensional vector space of global sections of $\mathcal{E}$. We define a map $\psi$ from $C$ to the space of $2 d \times 2 d$ skew-symmetric matrices with entries in the space of homogeneous polynomials of degree $d-1$, such that $\psi^{-1}\left(\mathcal{P}_{d}\right)=C$.

Choose a basis $\left\{s_{1}, \ldots, s_{2 d}\right\}$ for $U$ and define

$$
C \ni x \stackrel{\psi}{\mapsto} \sum_{1 \leq i<j \leq 2 d}\left(s_{i}(x) \wedge s_{j}(x)\right)\left(E_{i j}-E_{j i}\right)=\left[\begin{array}{ccc}
0 & & \\
\ddots & s_{i}(x) \wedge s_{j}(x) \\
& \ddots & \\
& & 0
\end{array}\right] .
$$

Since $s_{i} \wedge s_{j} \in \bigwedge^{2} U$, by property (iii) the map $\psi$ extends to

$$
\Psi: \mathbb{P}(E) \longrightarrow \mathbb{P}\left(\bigwedge^{2} U\right)
$$

given by a linear system of plane curves of degree $d-1$. In other words, $\Psi$ is a tensor in $S^{d-1}\left(E^{*}\right) \otimes\left(\bigwedge^{2} U\right)$. In coordinates it equals to a $2 d \times 2 d$ skew-symmetric matrix $B\left(x_{0}, x_{1}, x_{2}\right)$ with entries from the space of homogeneous polynomials of degree $d-1$.

From the definition of $B$ and isomorphism (3.1) in Lemma 3.1 it follows that at any point $x \in C \operatorname{rank} B(x)=2$. 
Before we proceed, we prove that a different basis $\left\{s_{1}^{\prime}, \ldots, s_{2 d}^{\prime}\right\}$ for $U$ induces equivalent representation $B^{\prime}$. Indeed, since

$$
\left[\begin{array}{c}
s_{1}^{\prime} \\
\vdots \\
s_{2 d}^{\prime}
\end{array}\right]=R \cdot\left[\begin{array}{c}
s_{1} \\
\vdots \\
s_{2 d}
\end{array}\right]
$$

for some constant invertible matrix $R$, it is straightforward to check that

$$
B^{\prime}=\left[\begin{array}{cccc}
0 & & & \\
& \ddots & s_{i}^{\prime} \wedge & s_{j}^{\prime} \\
& & \ddots & \\
& & 0
\end{array}\right]=R \cdot\left[\begin{array}{llll}
0 & & & \\
& \ddots & s_{i} \wedge s_{j} \\
& & \ddots & \\
& & & 0
\end{array}\right] \cdot R^{t}=R \cdot B \cdot R^{t}
$$

As before, let $F$ be the defining polynomial for $C$. Denote by $M$ a $4 \times 4$ submatrix of $B$, obtained by deleting $2 d-4$ rows and columns with the same indices. Then $F \mid \operatorname{Pf} M$ since rank $B(x)=2$ and therefore Pf $M(x)=0$ for all $x \in C$. Consider next a $6 \times 6$ skew-symmetric submatrix $N$ of $B$ and its Pfaffian adjoint $\tilde{N}$. By (3.2) we get

$$
\begin{array}{r}
\operatorname{Pf} N \cdot \operatorname{Pf} \tilde{N}=(\operatorname{Pf} N)^{3}, \\
\operatorname{Pf} \tilde{N}=(\operatorname{Pf} N)^{2} .
\end{array}
$$

The entries of $\tilde{N}$ are Pfaffians of $4 \times 4$ submatrices, hence $F^{3} \mid(\operatorname{Pf} N)^{2}$. Since $C$ is irreducible, $F^{2} \mid \operatorname{Pf} N$. By repeating this process we obtain that $F^{d-2}$ divides all the Pfaffians of $(2 d-2) \times(2 d-2)$ skew-symmetric submatrices of $B$. These are exactly $\operatorname{Pf}^{i j} B$ defined in the proof of Lemma 3.2. This means that

$$
A=\frac{1}{F^{d-2}} \tilde{B}
$$

is a matrix with entries in $E^{*}$. Since $\operatorname{rank} B(x)=2$ for all $x \in C$, we get rank $A(x)=2 d-2$. Therefore $\operatorname{Pf} A$ is a hypersurface of degree $d$ vanishing on $C$ unless $\operatorname{Pf} A$ is identically zero. This implies $\operatorname{Pf} A=c F$ for some constant $c$.

It remains to prove that $\operatorname{Pf} A \neq 0$. We consider the tautological map

$$
\sigma: \mathbb{P} \mathcal{E}^{\vee} \rightarrow \mathbb{P} U^{*}
$$

defined by the tautological linear system $\mathbb{H}=\left|\mathcal{O}_{\mathbb{P} \mathcal{E}}(1)\right|$. Choose $L$ such that it intersects $C$ in $d$ distinct points $\left\{p_{1}, \ldots, p_{d}\right\}$. The condition $H^{0}(C, \mathcal{E}(-1))=0$ is equivalent to the following property: no $H \in \mathbb{H}$ contains the union of the projective lines $\mathbb{P} \mathcal{E}_{p_{1}}^{\vee} \cup \ldots \cup \mathbb{P} \mathcal{E}_{p_{d}}^{\vee}$. This is equivalent to say that $\mathcal{E}_{p_{1}}^{\vee}, \ldots, \mathcal{E}_{p_{d}}^{\vee}$ are 2-dimensional subspaces of $U^{*}$ and their sum is $U^{*}$. There is no restriction to assume that $L$ 
is given by $z_{0}=0$. Using this equation in (3.5) we obtain the pencil of skewsymmetric matrices

$$
A_{L}=z_{1} A_{1}+z_{2} A_{2} .
$$

By fixing a basis for $U^{*}$ we can consider this as a pencil of matrices associated to a pencil of symplectic forms $z_{1} a_{1}+z_{2} a_{2}$ on $U^{*}$. By the preceding part of the proof it follows that $\mathcal{E}_{p_{i}}^{\vee}$ is the kernel of $z_{1}\left(p_{i}\right) a_{1}+z_{2}\left(p_{i}\right) a_{2}$. Since $h^{0}(C, \mathcal{E}(-1))=0$, it follows that

$$
U^{*}=\mathcal{E}_{p_{1}}^{\vee} \oplus \cdots \oplus \mathcal{E}_{p_{d}}^{\vee} .
$$

Hence we can take as a basis for $U^{*}$ the union of bases of the vector spaces $\mathcal{E}_{p_{i}}$. This basis gives the simultaneous reduction to the standard form of the skew-symmetric matrices of the pencil $A_{L}$. In this basis we have

$$
A_{L}=P^{t} J P,
$$

where $P$ is the change of basis matrix and

$$
J=\left[\begin{array}{cccc}
J_{1} & 0 & \cdots & 0 \\
0 & J_{2} & \cdots & 0 \\
\vdots & & \ddots & \vdots \\
0 & 0 & \cdots & 0
\end{array}\right]
$$

is the standard form. No $2 \times 2$ block $J_{i}$ is identically equal to 0 , since otherwise the rank of $A_{L}$ at $p_{i}$ would be at most $2 d-4$. Therefore $\operatorname{det} A_{L}$ is not identically zero, neither are $\operatorname{det} A$ and $\operatorname{Pf}(A)$.

By the classic result of Cook and Thomas [5, Theorem 1.1] two representations are equivalent if and only if the corresponding cokernels are isomorphic sheaves. Together with the above considerations it implies:

Theorem 3.5. There is a one to one correspondence between linear Pfaffian representations of $F$ (up to equivalence) and rank 2 bundles (up to isomorphism) on $C$ with the property

$$
\operatorname{det} \mathcal{E} \cong \mathcal{O}_{C}(d-1) \text { and } H^{0}(C, \mathcal{E}(-1))=0 .
$$

Proof. It remains to show that (i) in Proposition 3.4 follows from conditions (ii) and (iii). For every rank 2 bundle $\mathcal{E} \cong \mathcal{E}^{\vee} \otimes\left(\bigwedge^{2} \mathcal{E}\right.$ ) holds. Applying (iii) gives $\mathcal{E} \cong \mathcal{E}^{\vee} \otimes \mathcal{O}_{C}(d-1)$. Then

$$
\begin{array}{r}
H^{1}(C, \mathcal{E}(-1)) \\
\cong H^{1}\left(C, \mathcal{E}^{\vee} \otimes \mathcal{O}_{C}(d-2)\right) \cong H^{0}\left(C, \mathcal{E} \otimes \mathcal{O}_{C}(2-d) \otimes \mathcal{O}_{C}(d-3)\right) \\
\cong H^{0}(C, \mathcal{E}(-1))
\end{array}
$$

by Serre duality. Hence (ii) implies $H^{1}(C, \mathcal{E}(-1))=0$. 
Let $L$ be a section of $\mathcal{O}_{C}(1)$ and consider the exact sequence

$$
\left.0 \longrightarrow \mathcal{E}(-1) \longrightarrow \mathcal{E} \longrightarrow \mathcal{E}\right|_{L} \longrightarrow 0 .
$$

Note that $\left.\mathcal{E}\right|_{L}=\mathcal{E} \otimes \mathcal{O}_{L}$ is supported on a finite set of points. Taking cohomology gives a long exact sequence

$$
\begin{gathered}
H^{0}(C, \mathcal{E}(-1)) \rightarrow H^{0}(C, \mathcal{E}) \rightarrow H^{0}\left(C,\left.\mathcal{E}\right|_{L}\right) \rightarrow \\
0 \\
H^{1}(C, \mathcal{E}(-1)) \rightarrow H^{1}(C, \mathcal{E}) \rightarrow H^{1}\left(C,\left.\mathcal{E}\right|_{L}\right) \rightarrow \\
\| \\
0
\end{gathered}
$$

This proves that $H^{1}(C, \mathcal{E})=0$.

It also proves $h^{0}(C, \mathcal{E})=2 d$. Indeed, observe that $\left.\mathcal{E}\right|_{L}=\mathcal{E} \otimes \mathcal{O}_{L}$ is of rank 2 supported on the set $C \cap L$ of $d$ points, hence $h^{0}\left(C,\left.\mathcal{E}\right|_{L}\right)=2 d$.

Theorem 3.5 is a special case of the following Beauville's corollary, where representations of hypersurfaces are studied via arithmetically Cohen-Macaulay (ACM) sheaves on $\mathbb{P}^{n}$. One of the advantages of our elementary proof is an explicit construction of representations from sheaves.

Theorem 3.6 (Corollary 2.4 in [2]). Let $X$ be an integral hypersurface of degree $d$ in $\mathbb{P}^{n}\left(x_{0}, \ldots, x_{n}\right)$ over a field $k$ with char $k \neq 2$. Moreover, let $E$ be an ACM vector bundle on $X$ of rank 2 with determinant $\mathcal{O}_{X}(d+t)$. Then there exists a skewsymmetric matrix $A=\left(a_{i j}\right) \in \mathbb{M}_{l}$ with $a_{i j}$ homogeneous polynomials in $x_{0}, \ldots, x_{n}$ of degree $d_{i}+d_{j}-t$ and an exact sequence

$$
0 \rightarrow \bigoplus_{i=1}^{l} \mathcal{O}_{\mathbb{P}^{n}}\left(t-d_{i}\right) \stackrel{A}{\longrightarrow} \bigoplus_{i=1}^{l} \mathcal{O}_{\mathbb{P}^{n}}\left(d_{i}\right) \rightarrow E \rightarrow 0,
$$

where $X$ is defined by $\operatorname{Pf} A=0$.

If $H^{0}(X, E(-1))=0$ and $t=-1$, the entries of $A$ are linear.

To conclude this section we recall a result of Fujita [13, Example 6.4.16].

Remark 3.7. Suppose that $C$ has genus $g \geq 2$. If $\mathcal{E}$ is a bundle on $C$ such that $H^{1}(C, \mathcal{E})=0$, then $\mathcal{E}$ is ample. Being ample is equivalent to the condition that every quotient has strictly positive degree.

\section{The moduli space $M(2,2(g-1))$}

In this section we relate the set of Pfaffian representations to the moduli space of semistable vector bundles. 
As before let $C$ be a smooth plane curve of degree $d$ and genus $g$. The existence and properties of

$$
M_{C}(r, n),
$$

the moduli space of semistable vector bundles on $C$ of rank $r$ and degree $n$, were established in $[15,18]$ and more modern treatment can be found in [17]. It is known that $M_{C}(r, n)$ is an irreducible, normal projective variety with an open subset $M_{C}^{s}(r, n)$ corresponding to stable bundles.

If $C$ has genus $g \geq 2$ then $M_{C}^{s}(r, n)$ is not empty and its dimension is $r^{2}(g-1)+1$. The singular points of $M_{C}(r, n)$ are exactly $M_{C}(r, n) \backslash M_{C}^{s}(r, n)$.

If $C$ is an elliptic curve then $M_{C}^{s}(r, n)$ is empty.

One can restrict the study to the moduli space

$$
M_{C}(r, \mathcal{L})
$$

of (semistable) rank $r$ vector bundles on $C$ with determinant $\mathcal{L}$. As described in $[3,7]$ it is a closed subvariety in $M_{C}(r, \operatorname{deg} \mathcal{L})$. Moreover, $M_{C}^{s}(r, \mathcal{L})$ is a closed subvariety in $M_{C}^{s}(r, \operatorname{deg} \mathcal{L})$. The determinant can be fixed since the moduli space $M_{C}(r, \operatorname{deg} \mathcal{L})$ is, up to a finite étale covering, the product of $M_{C}(2, \mathcal{L})$ with the Jacobian $J C$.

Drezet and Narasimhan [7] showed that $\operatorname{Pic}\left(M_{C}(r, \mathcal{L})\right) \cong \mathbb{Z}$ is generated by geometrically defined Cartier divisors $\Theta_{r, \mathcal{L}}$ in $M_{C}(r, \mathcal{L})$. For example, when $\operatorname{deg} \mathcal{L}=r(g-1)$ then

$$
\chi(\mathcal{E})=0 \text { for all } \mathcal{E} \in M_{C}(r, \mathcal{L})
$$

and

$$
\Theta_{r, \mathcal{L}}=\left\{\mathcal{E} \in M_{C}(r, \mathcal{L}): h^{0}(C, \mathcal{E}) \neq 0\right\}
$$

is naturally such a divisor.

Theorem 4.1. Let $C$ be a smooth curve defined by a polynomial $F$ of degree $d$ in $\mathbb{P}^{2}$. There is a one to one correspondence between linear Pfaffian representations of $F$ (up to equivalence) and rank 2 bundles (up to isomorphism) on $C$ in the open set

$$
M_{C}\left(2, \mathcal{O}_{C}(d-3)\right) \backslash \Theta_{2, \mathcal{O}_{C}(d-3)} .
$$

Proof. From now on we consider the moduli space

$$
M_{C}\left(2, \mathcal{O}_{C}(d-3)\right)
$$

of (semistable) rank 2 vector bundles on $C$ with determinant $K_{C} \cong \mathcal{O}_{C}(d-3)$, which is a closed subvariety in $M_{C}(2, d(d-3))=M_{C}(2,2(g-1))$.

Recall from Theorem 3.5 the one to one correspondence between the linear Pfaffian representations of $C$ and rank 2 bundles on $C$ with property (3.6). For the 
sake of clearer notation, after tensoring by $\mathcal{O}_{C}(1)$, condition (3.6) can be rewritten into

$$
\operatorname{det} \mathcal{E} \cong \mathcal{O}_{C}(d-3) \text { and } H^{0}(C, \mathcal{E})=0 .
$$

Condition $h^{0}(C, \mathcal{E})=0$ implies that $\mathcal{E}$ is semistable. By the above considerations, bundles satisfying (4.1) can be parametrised by the points on

$$
M_{C}\left(2, \mathcal{O}_{C}(d-3)\right) \backslash \Theta_{2, \mathcal{O}_{C}(d-3)} .
$$

This is an open subset of $M_{C}\left(2, \mathcal{O}_{C}(d-3)\right)$ since we cut out a Cartier divisor.

\section{Pfaffians arising from decomposable vector bundles}

In this section we find and explicitly describe linear Pfaffian representations of $C$ (up to equivalence) arising from decomposable vector bundles. We parametrise them by an open set in the Kummer variety. Since decomposable vector bundles are never stable [9, Ex V.2.8], this open set lies in the singular locus of $M_{C}\left(2, \mathcal{O}_{C}(d-\right.$ $3)$ ). The proof of the fact that the moduli space of rank 2 bundles with canonical determinant is singular along the Kummer variety can be found in [16].

Kummer variety $\mathcal{K}_{C}$ of $C$ is by definition the quotient of the Jacobian $J C$ by the involution $\mathcal{L} \mapsto \mathcal{L}^{-1} \otimes \mathcal{O}_{C}(d-3)$.
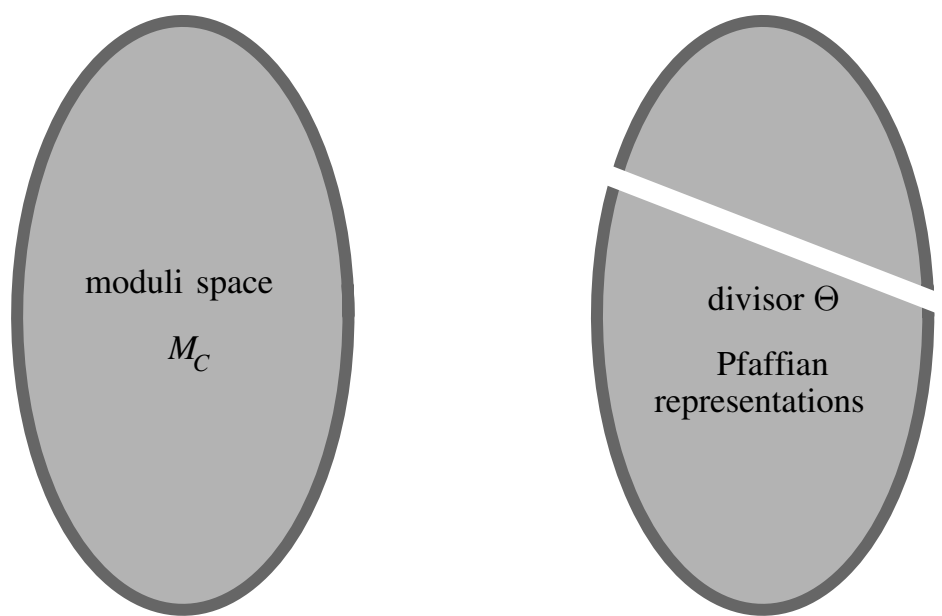

Figure 5.1. The dark boundaries represent the singular locus of $M_{C}$ and decomposable Pfaffians respectively.

Recall that by Theorem 3.5 and (4.1) we need to find all decomposable rank 2 bundles (up to isomorphism) with the property

$$
\operatorname{det} \mathcal{E} \cong \mathcal{O}_{C}(d-3) \text { and } H^{0}(C, \mathcal{E})=0 .
$$


Write $\mathcal{E}=\mathcal{L} \oplus \mathcal{M}$. Then $\operatorname{det} \mathcal{E} \cong \mathcal{L} \otimes \mathcal{M} \cong \mathcal{O}_{C}(d-3)$ or equivalently

$$
\mathcal{M} \cong \mathcal{L}^{-1} \otimes \mathcal{O}_{C}(d-3) .
$$

Observe that $\mathcal{E} \cong \mathcal{L} \oplus\left(\mathcal{L}^{-1} \otimes \mathcal{O}_{C}(d-3)\right)$ has no sections if and only if $\mathcal{L}$ and $\mathcal{L}^{-1} \otimes \mathcal{O}_{C}(d-3)$ have no sections. These can be calculated by the Riemann-Roch formula and Serre duality

$$
h^{0}(C, \mathcal{L})-h^{0}\left(C, \mathcal{L}^{-1} \otimes \mathcal{O}_{C}(d-3)\right)=\operatorname{deg} \mathcal{L}+1-g,
$$

since $\mathcal{O}_{C}(d-3)$ is exactly the line bundle of the canonical divisor $K_{C}$.

This proves that every decomposable rank 2 bundles with the property (4.1) is of the form

$$
\mathcal{E} \cong \mathcal{L} \oplus\left(\mathcal{L}^{-1} \otimes \mathcal{O}_{C}(d-3)\right)
$$

where $\mathcal{L}$ is a line bundle of degree $g-1=\frac{1}{2} d(d-3)$ with no sections. These are exactly the non exceptional line bundles defined in Section 2. We have seen that they correspond to the points on the Jacobian variety of $C$ not on the exceptional subvariety $W_{g-1}$.

Conversely, by Section 2 a point on the Jacobian variety induces a determinantal representation $M$ of $C$. Then

$$
\left[\begin{array}{cc}
0 & M \\
-M^{t} & 0
\end{array}\right]
$$

is a Pfaffian representation of $C$ with decomposable cokernel.

Thus we proved

Theorem 5.1. There is a one to one correspondence between decomposable vector bundles in $M_{C}\left(2, \mathcal{O}_{C}(d-3)\right) \backslash \Theta_{2, \mathcal{O}_{C}(d-3)}$ and the open subset of Kummer variety

$$
\left(J C \backslash W_{g-1}\right) / \equiv,
$$

where $\equiv$ is the involution $\mathcal{L} \mapsto \mathcal{L}^{-1} \otimes \mathcal{O}_{C}(d-3)$.

In the sequel we explicitly construct the above correspondence from the sections of $\mathcal{E}(1)$. As above, let $\mathcal{E}=\mathcal{L} \oplus\left(\mathcal{L}^{-1} \otimes \mathcal{O}_{C}(d-3)\right)$ with $\mathcal{L}$ of degree $g-1$ and no sections. Denote $\mathcal{L} \otimes \mathcal{O}_{C}(1)=\mathcal{L}(1)$ by $\mathcal{F}$. Then

$$
H^{0}(C, \mathcal{F}(-1))=0 \text { and } \operatorname{deg} \mathcal{F}=\frac{1}{2} d(d-1)=\operatorname{deg}\left(\mathcal{F}^{-1} \otimes \mathcal{O}_{C}(d-1)\right) .
$$

Let $H$ be a section of $\mathcal{O}_{C}(1)$ and consider the exact sequence

$$
\left.0 \longrightarrow \mathcal{F}(-1) \longrightarrow \mathcal{F} \longrightarrow \mathcal{F}\right|_{H} \longrightarrow 0
$$


where $\left.\mathcal{F}\right|_{H}$ is supported on a $d$ points $C \cap H$. Applying cohomology gives a long exact sequence with dimensions

$$
\begin{array}{ccc}
H^{0}(C, \mathcal{F}(-1)) & \rightarrow H^{0}(C, \mathcal{F}) \rightarrow H^{0}\left(C,\left.\mathcal{F}\right|_{H}\right) & \rightarrow H^{1}(C, \mathcal{F}(-1)) \rightarrow \\
0 & d & 0
\end{array}
$$

since $h^{0}(C, \mathcal{F}(-1))-h^{1}(C, \mathcal{F}(-1))=0$ by the Riemann-Roch theorem. This proves that $h^{0}(C, \mathcal{F})=d$. The same way we show $h^{0}\left(C, \mathcal{F}^{-1} \otimes \mathcal{O}_{C}(d-1)\right)=d$. Let $\left\{f_{1}, \ldots, f_{d}\right\}$ and $\left\{m_{1}, \ldots, m_{d}\right\}$ be bases of the complete linear system of $\mathcal{F}$ and $\mathcal{F}^{-1} \otimes \mathcal{O}_{C}(d-1)$ respectively. Then

$$
\left\{\left(f_{1}, 0\right), \ldots,\left(f_{d}, 0\right),\left(0, m_{1}\right), \ldots,\left(0, m_{d}\right)\right\}
$$

form a basis for $H^{0}(C, \mathcal{E}(1))$. Obviously $\left(f_{i}, 0\right) \wedge\left(0, m_{j}\right)=f_{i} \otimes m_{j}$, whereas $\left(f_{i}, 0\right) \wedge\left(f_{j}, 0\right)$ and $\left(0, m_{i}\right) \wedge\left(0, m_{j}\right)$ equal 0 . Applying the same argument as in the proof of Proposition 3.4, we see that the map $\Psi$ is of the form

$$
\left[\begin{array}{cc}
0 & f_{i} \otimes m_{j} \\
-f_{j} \otimes m_{i} & 0
\end{array}\right] .
$$

In coordinates it equals to a $2 d \times 2 d$ skew-symmetric matrix $B$ with entries from the space of homogeneous polynomials of degree $d-1$ and zero diagonal blocks. As in the proof of Proposition 3.4 we get that

$$
A=\frac{1}{F^{d-2}} \tilde{B}
$$

is a Pfaffian representation of $C$. In particular,

$$
A=\left[\begin{array}{cc}
0 & M \\
-M^{t} & 0
\end{array}\right],
$$

where $M$ is the determinantal representation of $C$ corresponding to the non exceptional line bundle $\mathcal{F}$.

\section{Cubic curves}

In this section $C$ will denote a curve defined by a smooth cubic polynomial $F$ in $\mathbb{P}^{2}$.

Corollary 6.1 ( $\$ 1$ in [3]). On a cubic curve $C$ all linear Pfaffian representations can be parametrised by the points on the Kummer variety $\mathcal{K}_{C}-\{$ one point $\}$. 
Proof. Recall that on an elliptic curve $K_{C} \cong \mathcal{O}_{C}$. Since $M_{C}^{s}(2,0)$ is empty, there are no stable bundles on $C$. On the other hand, by [3, §4] the non-stable part of $M_{C}\left(2, \mathcal{O}_{C}\right)$ consists of decomposable vector bundles of the form $\mathcal{L} \oplus \mathcal{L}^{-1}$ for $\mathcal{L}$ in the Jacobian $J C$. Obviously $\mathcal{L} \oplus \mathcal{L}^{-1}$ and $\mathcal{L}^{-1} \oplus \mathcal{L}$ are equivalent. For $\mathcal{L} \in J C$ the following conditions are equivalent:

- $h^{0}\left(C, \mathcal{L} \oplus \mathcal{L}^{-1}\right)=0$,

- $h^{0}(C, \mathcal{L})=0$,

- $\mathcal{L} \neq \mathcal{O}_{C}$.

Therefore

$$
M_{C}\left(2, \mathcal{O}_{C}\right) \backslash \Theta_{2, \mathcal{O}_{C}}=\left\{\mathcal{L} \oplus \mathcal{L}^{-1} ; \mathcal{L} \in J C\right\} \backslash\left\{\mathcal{O}_{C} \oplus \mathcal{O}_{C}\right\}
$$

Vinnikov [22] found an explicit one to one correspondence between the linear determinantal representations (up to equivalence) of $C$ and the points on an affine piece of $C$ :

Lemma 6.2 ( [22]). Every smooth cubic can be brought into the Weierstrass form

$$
F\left(x_{0}, x_{1}, x_{2}\right)=-x_{1} x_{2}^{2}+x_{0}^{3}+\alpha x_{0} x_{1}^{2}+\beta x_{1}^{3} .
$$

A complete set of determinantal representations of $F$ is

$$
x_{0} \mathrm{Id}+x_{2}\left(\begin{array}{lll}
0 & 1 & 0 \\
0 & 0 & 1 \\
0 & 0 & 0
\end{array}\right)+x_{1}\left(\begin{array}{ccc}
\frac{s}{2} & l & \alpha+\frac{3}{4} s^{2} \\
0 & -s & -l \\
-1 & 0 & \frac{s}{2}
\end{array}\right),
$$

where $l^{2}=s^{3}+\alpha s+\beta$. Note that the last equation is exactly the affine part $F(s, 1, l)$.

Recall that the Jacobian of a cubic curve $C$ with $g=1$ is the curve itself and $J-\left\{W_{0}\right\}$ is an affine piece of $C$. In particular, Corollary 6.1 implies that the complete set of Pfaffian representations of $F$ (put in the Weierstrass form) equals

$$
\left[\begin{array}{cc}
0 & M \\
-M^{t} & 0
\end{array}\right]
$$

where $M$ are the determinantal representations in Lemma 6.2. Note that $M$ and $-M^{t}$ are not equivalent determinantal representations, but

$$
\left[\begin{array}{cc}
0 & M \\
-M^{t} & 0
\end{array}\right] \text { and }\left[\begin{array}{cc}
0 & -M^{t} \\
M & 0
\end{array}\right]
$$

are quivalent Pfaffian representations since

$$
\left[\begin{array}{ll}
0 & I \\
I & 0
\end{array}\right]\left[\begin{array}{cc}
0 & M \\
-M^{t} & 0
\end{array}\right]\left[\begin{array}{ll}
0 & I \\
I & 0
\end{array}\right]=\left[\begin{array}{cc}
0 & -M^{t} \\
M & 0
\end{array}\right] .
$$




\section{Examples of higher genus}

We start by an example of a genus 3 curve.

Example 7.1. Any non hyperelliptic curve $C$ of genus 3 is isomorphic to a plane quartic. In this case $M_{C}\left(2, \mathcal{O}_{C}(1)\right) \cong M_{C}\left(2, \mathcal{O}_{C}\right)$ embeds as a Coble quartic hypersurface in $\mathbb{P}^{7}$ and is singular along the Kummer variety $\mathcal{K}_{C}$. For references check $[3,12,14]$.

For a given plane quartic Vanhaecke [21] gives explicit equations of the Coble quartic hypersurface. First he finds the equations of the Kummer variety which represents the singular locus of the moduli space, from here the Coble quartic is obtained by integration. For example, the moduli space of

$$
C: x^{4}-y z^{3}-y^{4}=0
$$

is the Coble hypersurface in $\mathbb{P}^{7}$ defined by the polynomial

$$
\begin{aligned}
& z_{0}^{4}-z_{2}^{4}-2 z_{1} z_{2}^{2} z_{3}-z_{1}^{2} z_{3}^{2}-z_{0} z_{3}^{3}-4 z_{0} z_{2}^{2} z_{4}-2 z_{0}^{2} z_{4}^{2}-3 z_{0} z_{2} z_{4}^{2} \\
+ & z_{4}^{4}-4 z_{0} z_{1} z_{2} z_{5}+z_{3} z_{4} z_{5}^{2}+z_{2} z_{5}^{3}-4 z_{0} z_{1}^{2} z_{6}-3 z_{0}^{2} z_{3} z_{6}-z_{3} z_{4}^{2} z_{6}-2 z_{2} z_{4} z_{5} z_{6} \\
+ & z_{1} z_{5}^{2} z_{6}-z_{0} z_{6}^{3}-2 z_{0}^{2} z_{1} z_{7}-z_{3}^{2} z_{4} z_{7}+2 z_{1} z_{4}^{2} z_{7}-z_{2} z_{3} z_{5} z_{7} \\
+ & z_{0} z_{5}^{2} z_{7}-z_{2}^{2} z_{6} z_{7}-z_{1} z_{3} z_{6} z_{7}-4 z_{0} z_{4} z_{6} z_{7}+z_{1}^{2} z_{7}^{2}
\end{aligned}
$$

In the sequel we outline an algorithm for finding all Pfaffian representations of the given $C$ (up to equivalence) based on canonical forms of matrix pairs. This is a generalisation of Vinnikov's construction of determinantal representations [22]. Let $A=x A_{x}+z A_{z}+y A_{y}$ be a Pfaffian representation of $C$. Observe that $A_{x}$ is invertible and $A_{z}$ nilpotent since $C$ is defined by Pf $A$ and contains $x^{4}$ term and no $z^{4}$ term. Because $C$ contains also $y z^{3}$, by Lancaster and Rodman [11, Theorem 5.1] every Pfaffian representation of $C$ can be put into the skew-symmetric canonical form

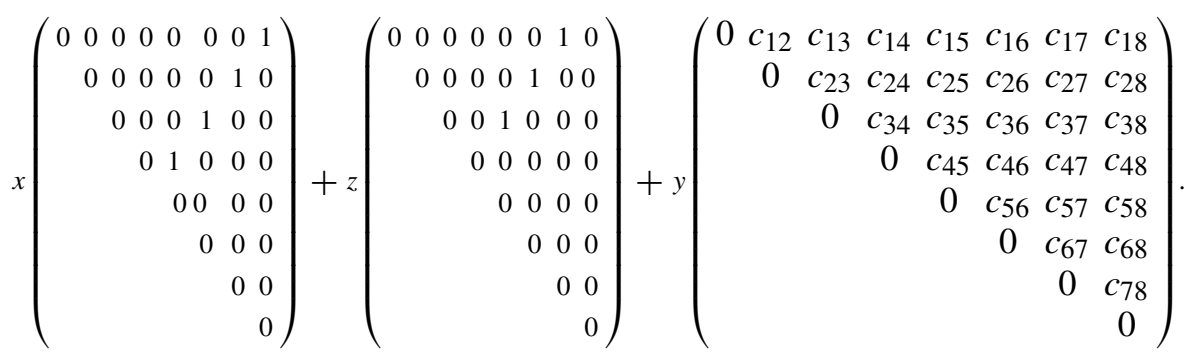


Since Pf $A$ equals the equation of $C$ we get

$$
\begin{aligned}
& c_{45}=-c_{18}-c_{36}-c_{27}, \\
& c_{46}=-c_{28}-c_{37}, \\
& c_{47}=-c_{38}, \\
& c_{48}=1, \\
& c_{15}=c_{18}\left(c_{18}-c_{27}\right)-c_{27}^{2}+c_{28} c_{35}-\left(c_{18}+c_{27}+c_{36}\right) c_{36}-c_{26} c_{37}-\ldots, \\
& c_{16}=-c_{25}-2\left(c_{18}+c_{27}\right) c_{28}-\left(c_{18}+2 c_{27}+c_{36}\right) c_{37}+c_{35} c_{38}+c_{34} c_{57}+\ldots, \\
& c_{26}=-c_{17}-c_{28}^{2}-c_{35}-c_{28} c_{37}-\left(2 c_{18}+c_{27}+c_{36}\right) c_{38}+c_{34} c_{58}+c_{24} c_{68}+c_{14} c_{78} .
\end{aligned}
$$

There are 21 parameters $c_{i j}$ left in the representation. Pfaffian representations are equivalent under the action

$$
A \mapsto P \cdot A \cdot P^{t},
$$

where $P$ is an invertible constant matrix. By a suitable $P$ we can reduce the number of parameters in $A$. In other words, we will reduce the number of equivalent representations in each equivalence class.

Lemma 7.2. The action $A \mapsto P \cdot A \cdot P^{t}$ preserves the canonical form of the first two matices in the representation if and only if $P$ equals

$$
\left[\begin{array}{lc}
P_{1} & P_{2} \\
P_{3} & P_{1}^{-1}+P_{3} P_{1}^{-1} P_{2}
\end{array}\right] \text { or }\left[\begin{array}{ccc}
P_{2} & P_{1} \\
-P_{1}^{-1}+P_{3} P_{1}^{-1} P_{2} & P_{3}
\end{array}\right]
$$

where $P_{1}$ is invertible and $P_{i}$ are of the form

$$
\left[\begin{array}{cccc}
p_{i 1} & p_{i 2} & p_{i 3} & p_{i 4} \\
0 & p_{i 1} & p_{i 2} & p_{i 3} \\
0 & 0 & p_{i 1} & p_{i 2} \\
0 & 0 & 0 & p_{i 1}
\end{array}\right], \quad i=1,2,3
$$

Proof. Denote

$$
I=\left[\begin{array}{llll}
0 & 0 & 0 & 1 \\
0 & 0 & 1 & 0 \\
0 & 1 & 0 & 0 \\
1 & 0 & 0 & 0
\end{array}\right] \text { and } N=\left[\begin{array}{llll}
0 & 0 & 1 & 0 \\
0 & 1 & 0 & 0 \\
1 & 0 & 0 & 0 \\
0 & 0 & 0 & 0
\end{array}\right]
$$

We will need the following obvious observation, which can be proved directly by comparing matrix elements:

Let $Y, Y^{\prime}$ be $8 \times 8$ matrices for which

$$
Y \cdot\left[\begin{array}{cc}
0 & I \\
-I & 0
\end{array}\right]=\left[\begin{array}{cc}
0 & I \\
-I & 0
\end{array}\right] \cdot Y^{\prime} \text { and } Y \cdot\left[\begin{array}{cc}
0 & N \\
-N & 0
\end{array}\right]=\left[\begin{array}{cc}
0 & N \\
-N & 0
\end{array}\right] \cdot Y^{\prime} \text { hold. }
$$


Then $Y^{\prime}=Y^{t}$ and $Y=\left[\begin{array}{ll}Y_{1} & Y_{2} \\ Y_{3} & Y_{4}\end{array}\right]$, where

$$
Y_{i}=\left[\begin{array}{cccc}
y_{i 1} & y_{i 2} & y_{i 3} & y_{i 4} \\
0 & y_{i 1} & y_{i 2} & y_{i 3} \\
0 & 0 & y_{i 1} & y_{i 2} \\
0 & 0 & 0 & y_{i 1}
\end{array}\right], i=1,2,3,4
$$

We call the specific form of the above Toeplitz matrices " $\triangle$ form".

Now we can find all invertible

$$
P=\left[\begin{array}{ll}
P_{1} & P_{2} \\
P_{3} & P_{4}
\end{array}\right]
$$

that satisfy

$$
P \cdot\left[\begin{array}{cc}
0 & I \\
-I & 0
\end{array}\right] \cdot P^{t}=\left[\begin{array}{cc}
0 & I \\
-I & 0
\end{array}\right] \text { and } P \cdot\left[\begin{array}{cc}
0 & N \\
-N & 0
\end{array}\right] \cdot P^{t}=\left[\begin{array}{cc}
0 & N \\
-N & 0
\end{array}\right] .
$$

By the above observation all $P_{i}$ 's are of $\triangle$ form. Moreover, if $P_{1}$ is invertible then $P_{4}=P_{1}^{-1}+P_{3} P_{1}^{-1} P_{2}$. The same way we see that $P_{3}=-P_{2}^{-1}+P_{1} P_{2}^{-1} P_{4}$ when $P_{2}$ is invertible.

Since $P$ is invertible and consists of $\triangle$ blocks, at least one of $P_{1}, P_{2}$ is also invertible. Note that

$$
\left[\begin{array}{ll}
P_{1} & P_{2} \\
P_{3} & P_{4}
\end{array}\right] \cdot\left[\begin{array}{cc}
0 & -\mathrm{Id} \\
\mathrm{Id} & 0
\end{array}\right]=\left[\begin{array}{ll}
P_{2} & -P_{1} \\
P_{4} & -P_{3}
\end{array}\right]
$$

exchanges $P_{1}$ and $P_{2}$ which finishes the proof.

The action of Lemma 7.2 enables us to reduce the number of parameters $c_{i j}$. We can choose such $P$ that its action eliminates

$$
\begin{array}{r}
c_{14}=c_{24}=c_{34}=c_{38}=c_{47}=0, \\
c_{18}=c_{45}=-\frac{1}{2}\left(c_{27}+c_{36}\right), c_{28}=c_{46}=-\frac{1}{2} c_{37} .
\end{array}
$$

This computation can be easily checked by using Wolfram Mathematica.

The relations among $c_{i j}$ then simplify to:

$$
\begin{aligned}
c_{15} & =\frac{1}{4}\left(-2\left(c_{27}+c_{36}\right)^{2}-\left(c_{27}-c_{36}\right)^{2}+6\left(c_{17}+c_{35}\right) c_{37}-c_{37}^{3}+4 c_{23} c_{67}\right), \\
c_{16}+c_{25} & =-\left(c_{27}+c_{36}\right) c_{37} \\
c_{26} & =-\left(c_{17}+c_{35}\right)+c_{37}^{2} / 4
\end{aligned}
$$


and

$$
\begin{aligned}
& c_{27}^{3}+4 c_{16} c_{35}+c_{36}^{3}+4 c_{26}\left(c_{27}+c_{36}\right) c_{37}+c_{27} c_{35} c_{37} \\
&+c_{17}\left(4 c_{25}-\left(c_{27}-c_{36}\right) c_{37}\right)+4 c_{13} c_{56}+4 c_{12} c_{57} \\
&=-2 c_{23} c_{37} c_{57}-2 c_{13} c_{37} c_{67}+c_{27} c_{36}\left(c_{27}+c_{36}\right)+c_{35} c_{36} c_{37} \\
&+2\left(c_{16}+c_{25}\right) c_{37}^{2}+4\left(c_{27}+c_{36}\right)\left(c_{23} c_{67}+c_{15}\right), \\
& 4 c_{15} c_{27} c_{36}+c_{26} c_{27}^{2} c_{37}+c_{27}^{2} c_{35} c_{37}+2 c_{26} c_{27} c_{36} c_{37}+c_{27} c_{35} c_{36} c_{37}+c_{26} c_{36}^{2} c_{37}+c_{15} c_{37}^{3}+4 c_{12} c_{37} c_{56} \\
&+c_{17}\left(4 c_{26} c_{35}+c_{27} c_{36} c_{37}+c_{36}^{2} c_{37}+4 c_{23} c_{56}\right) \\
&+4 c_{13} c_{26} c_{57}-c_{27} c_{36}\left(c_{27}+c_{36}\right)^{2}-4 c_{23} c_{27} c_{37} c_{57}+c_{23} c_{36} c_{37} c_{57} \\
&+4 c_{15} c_{23} c_{67}+4 c_{12} c_{35} c_{67}+c_{13} c_{27} c_{37} c_{67}+c_{13} c_{36} c_{37} c_{67} \\
&= 4 c_{15} c_{26} c_{37}+c_{25}\left(c_{27}+c_{36}\right) c_{37}^{2}+4 c_{13} c_{27} c_{56}+c_{17}\left(4 c_{25} c_{36}+c_{35} c_{37}^{2}\right) \\
&+4 c_{12} c_{36} c_{57}+c_{13} c_{37}^{2} c_{57}+c_{16}\left(4 c_{27} c_{35}-4 c_{25} c_{37}+c_{27} c_{37}^{2}+c_{36} c_{37}^{2}\right. \\
&\left.+4 c_{23} c_{57}\right)+4 c_{13} c_{25} c_{67}+c_{23}\left(c_{27}+c_{36}\right)^{2} c_{67}, \\
& 4 c_{16} c_{27}+4 c_{25} c_{36}+4 c_{15} c_{37}+c_{35} c_{37}^{2}+c_{17}\left(-4 c_{26}-4 c_{35}+c_{37}^{2}\right) \\
&= 4 c_{26} c_{35}+2 c_{27}^{2} c_{37}+4 c_{27} c_{36} c_{37}+2 c_{36}^{2} c_{37}+4 c_{23} c_{56}+4 c_{13} c_{57}+4 c_{12} c_{67} .
\end{aligned}
$$

We are left with 12 parameters

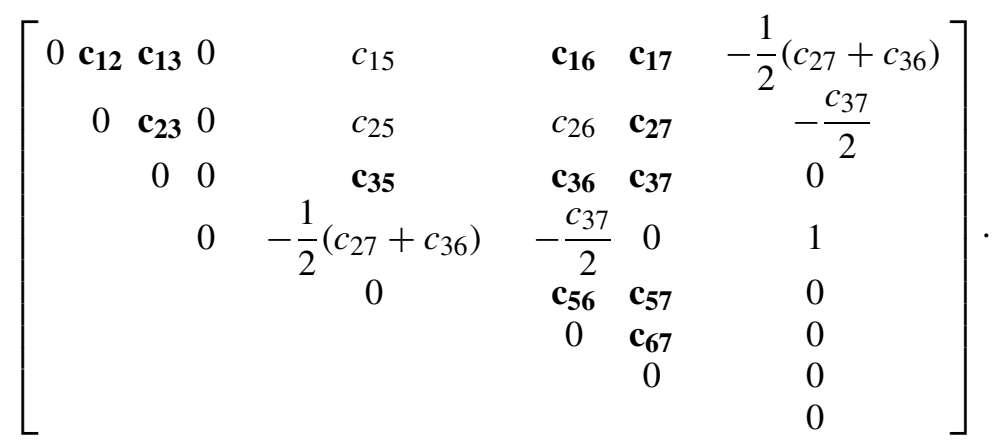

Note that we have chosen $P$ to make the top right $4 \times 4$ corner as symmetric as possible. We will show in the proof of Lemma 7.3 that the change of coordinates

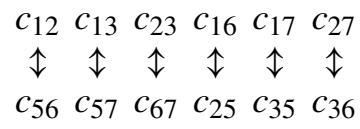

in (7.1) and (7.2) yields an equivalent representation.

Next we prove that the equivalence class of a generic Pfaffian representation above is 3 dimensional. 
Lemma 7.3. Pfaffian representations

$$
\begin{aligned}
& A=x\left[\begin{array}{cc}
0 & I \\
-I & 0
\end{array}\right]+z\left[\begin{array}{cc}
0 & N \\
-N & 0
\end{array}\right]+y\left[c_{i j}\right] \text { and } \\
& A^{\prime}=x\left[\begin{array}{cc}
0 & I \\
-I & 0
\end{array}\right]+z\left[\begin{array}{cc}
0 & N \\
-N & 0
\end{array}\right]+y\left[c_{i j}^{\prime}\right]
\end{aligned}
$$

with $\left[c_{i j}\right],\left[c_{i j}^{\prime}\right]$ in the form (7.2) are equivalent if and only if

$$
\begin{gathered}
{\left[\begin{array}{cccc}
c_{12}^{\prime} & c_{13}^{\prime} & c_{23}^{\prime} \\
c_{16}^{\prime}-c_{25}^{\prime} & c_{17}^{\prime}-c_{35}^{\prime} & c_{27}^{\prime}-c_{36}^{\prime} \\
c_{56}^{\prime} & c_{57}^{\prime} & c_{67}^{\prime} \\
c_{16}^{\prime}+c_{25}^{\prime} & c_{17}^{\prime}+c_{35}^{\prime} & c_{27}^{\prime}+c_{36}^{\prime}
\end{array}\right]} \\
\mapsto\left[\begin{array}{cccc}
p_{1}^{2} & p_{1} p_{2} & p_{2}^{2} & 0 \\
2 p_{1} p_{3} & p_{2} p_{3}+p_{1} p_{4} & 2 p_{2} p_{4} & 0 \\
p_{3}^{2} & p_{3} p_{4} & p_{4}^{2} & 0 \\
0 & 0 & 0 & 1
\end{array}\right] \cdot\left[\begin{array}{ccc}
c_{12} & c_{13} & c_{23} \\
c_{16}-c_{25} & c_{17}-c_{35} & c_{27}-c_{36} \\
c_{56} & c_{57} & c_{67} \\
c_{16}+c_{25} & c_{17}+c_{35} & c_{27}+c_{36}
\end{array}\right],
\end{gathered}
$$

for some $p_{1}, p_{2}, p_{3}, p_{4} \in k$ which satisfy $1+p_{2} p_{3}=p_{1} p_{4}$.

Proof. An action $A \mapsto P \cdot A \cdot P^{t}$ with invertible $P_{1}$ preserves all 0 and 1 elements in the above matrix if and only if

$$
P_{i}=\left[\begin{array}{cccc}
p_{i} & 0 & 0 & 0 \\
0 & p_{i} & 0 & 0 \\
0 & 0 & p_{i} & 0 \\
0 & 0 & 0 & p_{i}
\end{array}\right], \quad i=1,2,3
$$

This can be checked by a straightforward calculation using Lemma 7.2. Moreover, $c_{27}+c_{36}, c_{17}+c_{35}, c_{37}$ and therefore also $c_{16}+c_{25}, c_{15}, c_{26}$ are invariant under this action. The action on the remaining parameters can be neatly written in the following matrix form

$$
\begin{aligned}
& {\left[\begin{array}{ccc}
c_{12} & c_{13} & c_{23} \\
c_{16}-c_{25} & c_{17}-c_{35} & c_{27}-c_{36} \\
c_{56} & c_{57} & c_{67}
\end{array}\right]} \\
& \mapsto\left[\begin{array}{ccc}
p_{1}^{2} & p_{1} p_{2} & p_{2}^{2} \\
2 p_{1} p_{3} & 2 p_{2} p_{3}+1 & 2 \frac{p_{2}}{p_{1}}\left(1+p_{2} p_{3}\right) \\
p_{3}^{2} & \frac{p_{3}}{p_{1}}\left(1+p_{2} p_{3}\right) & \frac{1}{p_{1}^{2}}\left(1+p_{2} p_{3}\right)^{2}
\end{array}\right] \cdot\left[\begin{array}{ccc}
c_{12} & c_{13} & c_{23} \\
c_{16}-c_{25} & c_{17}-c_{35} & c_{27}-c_{36} \\
c_{56} & c_{57} & c_{67}
\end{array}\right] .
\end{aligned}
$$


Note that $P\left(p_{1}, p_{2}, p_{3}\right)$ has determinant 1 and that $c_{16} c_{25}+c_{12} c_{56}, c_{17} c_{35}+$ $c_{13} c_{57}, c_{27} c_{36}+c_{23} c_{67}$ is another set of invariants.

When $P_{1}$ is not invertible it is (by the proof of Lemma 7.2) enough to consider

$$
\begin{aligned}
& {\left[\begin{array}{cc}
0 & -\mathrm{Id} \\
\mathrm{Id} & 0
\end{array}\right] \cdot\left[c_{i j}\right] \cdot\left[\begin{array}{cc}
0 & \mathrm{Id} \\
-\mathrm{Id} & 0
\end{array}\right]=}
\end{aligned}
$$

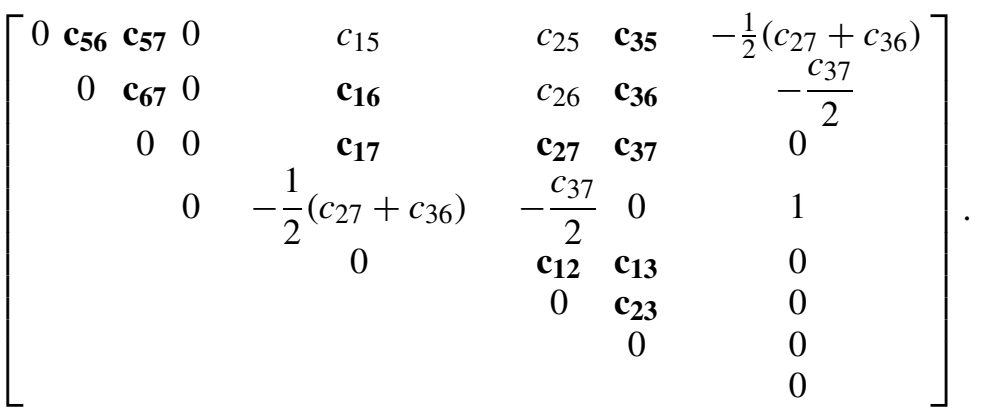

which also preserves all 0 and 1 elements in (7.2). As before

$$
\begin{array}{r}
c_{27}+c_{36}, c_{17}+c_{35}, c_{37}, \\
c_{16}+c_{25}, c_{15}, c_{26}, \\
c_{16} c_{25}+c_{12} c_{56}, c_{17} c_{35}+c_{13} c_{57}, c_{27} c_{36}+c_{23} c_{67}
\end{array}
$$

are invariants and

$$
\begin{gathered}
{\left[\begin{array}{ccc}
c_{12} & c_{13} & c_{23} \\
c_{16}-c_{25} & c_{17}-c_{35} & c_{27}-c_{36} \\
c_{56} & c_{57} & c_{67}
\end{array}\right]} \\
\mapsto\left[\begin{array}{ccc}
0 & 0 & 1 \\
0 & -1 & 0 \\
1 & 0 & 0
\end{array}\right] \cdot\left[\begin{array}{ccc}
c_{12} & c_{13} & c_{23} \\
c_{16}-c_{25} & c_{17}-c_{35} & c_{27}-c_{36} \\
c_{56} & c_{57} & c_{67}
\end{array}\right] \\
=\left[\begin{array}{ccc}
c_{56} & c_{57} & c_{67} \\
-c_{16}+c_{25} & -c_{17}+c_{35} & -c_{27}+c_{36} \\
c_{12} & c_{13} & c_{23}
\end{array}\right] .
\end{gathered}
$$

We reduced the description of our representation to 12 parameters $c_{i j}$ modulo 3 dimensional $P\left(p_{1}, p_{2}, p_{3}, p_{4}\right)$ action. This proves that all Pfaffian representations of $C$ are $6=12-3$ (action of $P$ ) -3 (relations in (7.1) among $c_{i j}$ ) dimensional. As described in Theorem 4.1 they corresponds to the open set $M_{C}\left(2, \mathcal{O}_{C}(1)\right) \backslash \Theta_{2, \mathcal{O}_{C}(1)}$.

Remark 7.4. Using local parameters and implicit function theorem, it is easy to see that

$$
c_{12}=c_{13}=c_{23}=c_{56}=c_{57}=c_{67}=0
$$


is the singular locus of the space of all Pfaffian representations of $C$. Note that these representations are decomposable and non-equivalent to each other. In the next paragraph will show that every such representation arises from a decomposable vector bundle (as expected). These vector bundles correspond to an open subset in the 3 dimensional Kummer variety.

By Theorem 5.1 there exists a one to one correspondence between the decomposable vector bundles in $M_{C}\left(2, \mathcal{O}_{C}(1)\right) \backslash \Theta_{2, \mathcal{O}_{C}(1)}$ and the open subset of Kummer variety

$$
\text { (Jacobian variety of } \left.C \backslash W_{2}\right) / \equiv \text {, }
$$

where $\equiv$ is the involution $\mathcal{L} \mapsto \mathcal{L}^{-1} \otimes \mathcal{O}_{C}(1)$. Vinnikov in [22] explicitely parametrised the set of all determinantal representations of $C$ (up to equivalence) by the points on the Jacobian variety without the exceptional subvariety $W_{2}$.

Thus we start by finding all determinantal representations of $C$ up to equivalence. Write $M(x, y, z)=x M_{x}+y M_{y}+z M_{z}$ with $\operatorname{det} M=x^{4}-y z^{3}-y^{4}$. The action $M \mapsto M_{x}^{-1} \cdot M$ maps to an equivalent representation since $\operatorname{det} M_{x} \neq 0$. Additionally, since det $M_{z}=0$ and det $M$ contains $z^{3}$ term, $M \mapsto R \cdot M \cdot R^{-1}, R \in$ $\mathrm{GL}_{4}(k)$ preserves $M_{x}=\mathrm{Id}$ and sets

$$
M_{z}=\left[\begin{array}{llll}
0 & 1 & 0 & 0 \\
0 & 0 & 1 & 0 \\
0 & 0 & 0 & 1 \\
0 & 0 & 0 & 0
\end{array}\right]
$$

Repeat the proof of Lemma 7.2 to show that $M \mapsto R \cdot M \cdot S, R, S \in \mathrm{GL}_{4}(k)$ preserves $M_{x}$ and $M_{z}$ if and only if $S=R^{-1}$ and is of $\triangle$ form.

We can choose such $R$ that its action reduces $M$ to the following form

$$
x \mathrm{Id}+z\left[\begin{array}{llll}
0 & 1 & 0 & 0 \\
0 & 0 & 1 & 0 \\
0 & 0 & 0 & 1 \\
0 & 0 & 0 & 0
\end{array}\right]+y\left[\begin{array}{cccc}
-\frac{1}{2}\left(c_{27}+c_{36}\right) & c_{17} & c_{16} & c_{15} \\
-\frac{c_{37}}{2} & c_{27} & c_{26} & c_{25} \\
0 & c_{37} & c_{36} & c_{35} \\
1 & 0 & -\frac{c_{37}}{2} & -\frac{1}{2}\left(c_{27}+c_{36}\right)
\end{array}\right]
$$

where

$$
\begin{aligned}
c_{15} & =\frac{1}{4}\left(-2\left(c_{27}+c_{36}\right)^{2}-\left(c_{27}-c_{36}\right)^{2}+6\left(c_{17}+c_{35}\right) c_{37}-c_{37}^{3}\right) \\
c_{16}+c_{25} & =-\left(c_{27}+c_{36}\right) c_{37} \\
c_{26} & =-\left(c_{17}+c_{35}\right)+c_{37}^{2} / 4
\end{aligned}
$$


and

$$
\begin{aligned}
& 2\left(c_{16}-c_{25}\right)\left(c_{17}-c_{35}\right)-4\left(c_{27}+c_{36}\right)\left(c_{27}^{2}+c_{36}^{2}\right) \\
& =-\left(13 c_{17} c_{27}+11 c_{27} c_{35}+11 c_{17} c_{36}+13 c_{35} c_{36}\right) c_{37}+4\left(c_{27}+c_{36}\right) c_{37}^{3} \text {, } \\
& 2(c 16-c 25)(c 27-c 36)+4\left(c 17^{2}+c 17 c 35+c 35^{2}\right) \\
& =\left(7 c 27^{2}+10 c 27 c 36+7 c 36^{2}\right) c 37-6(c 17+c 35) c 37^{2}+c 37^{4}, \\
& -4\left(c_{16}-c_{25}\right)^{2} c_{37}-16+8 c_{17}^{3}+8 c_{35}^{3}+8 c_{27}^{4}+8 c_{36}^{4} \\
& =8\left(\left(c_{17}+c_{35}\right)\left(5 c_{27}^{2}+6 c_{27} c_{36}+5 c_{36}^{2}\right)+2\left(c_{17} c_{27}^{2}+c_{35} c_{36}^{2}\right)\right) c_{37} \\
& -36\left(c_{17}+c_{35}\right)^{2} c_{37}^{2}-\left(17 c_{27}^{2}+30 c_{27} c_{36}+17 c_{36}^{2}\right) c_{37}^{3}+6\left(c_{17}+c_{35}\right) c_{37}^{4},
\end{aligned}
$$

which is the same as (7.1) for $c_{12}=c_{13}=c_{23}=c_{56}=c_{57}=c_{67}=0$. Here we chose parameters $c_{i j}$ to be compatible with the Pfaffian representations considered above. Indeed, if we multiply $M$ by

$$
\left[\begin{array}{llll}
0 & 0 & 0 & 1 \\
0 & 0 & 1 & 0 \\
0 & 1 & 0 & 0 \\
1 & 0 & 0 & 0
\end{array}\right]
$$

and fold it into the skew-symmetric block matrix, then

$$
\begin{aligned}
& {\left[\begin{array}{cc}
0 & M \\
-M^{t} & 0
\end{array}\right]=x\left[\begin{array}{cc}
0 & I \\
-I & 0
\end{array}\right]+z\left[\begin{array}{cc}
0 & N \\
-N & 0
\end{array}\right]}
\end{aligned}
$$

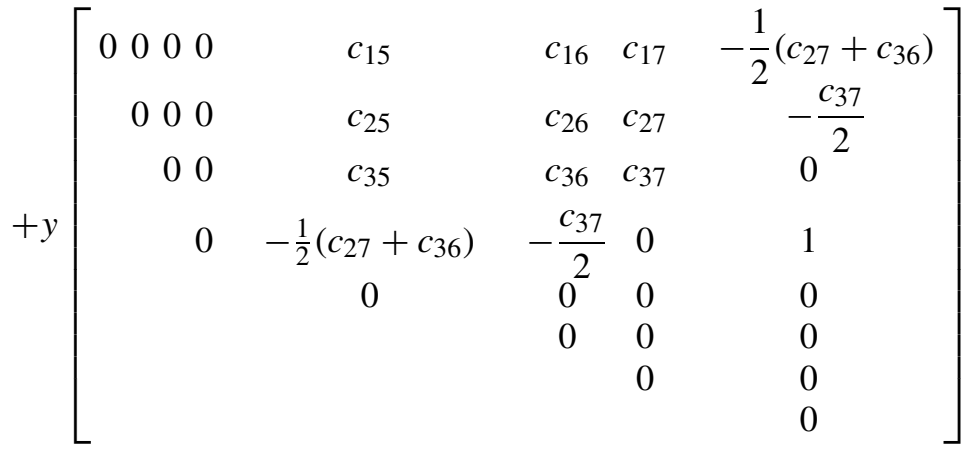

is the set of all decomposable Pfaffian representations of $C$. The set has dimension $3=6\left(\right.$ parameters $\left.c_{16}, c_{17}, c_{27}, c_{35}, c_{36}, c_{37}\right)-3$ (relations (7.3)). This is compatible with Remark 7.4. It is easy to show that $\operatorname{Ker} M$ is a line bundle of degree 6 . Then $\mathcal{L}=\operatorname{Ker} M \otimes \mathcal{O}_{C}(-1)$ and $\mathcal{L}_{t}=\operatorname{Ker} M^{t} \otimes \mathcal{O}_{C}(-1)$ both have degree 2 since $\operatorname{deg} \mathcal{O}_{C}(1)=4$. Moreover,

$$
\mathcal{L} \oplus \mathcal{L}_{t} \in M_{C}\left(2, \mathcal{O}_{C}(1)\right) \backslash \Theta_{2, \mathcal{O}_{C}(1)}
$$


and

$$
\mathcal{L}_{t} \cong \mathcal{L}^{-1} \otimes \mathcal{O}_{C}(1)
$$

which explains the involution in the Kummer variety.

We conclude the example by suggesting that explicit descriptions of the moduli space could be used to prove its rationality.

Let $C$ be a generic smooth plane curve of genus $g \geq 3$. By [16] the moduli space $M_{C}\left(2, \mathcal{O}_{C}(d-3)\right)$ of rank 2 bundles with canonical determinant embeds into $|2 \Theta|$. Its singular locus is isomorphic to the Kummer variety. Moreover, the embedding restricts to the Kummer map on the singular locus.

There are few results in the literature explicitly describing the above moduli spaces for smooth curves of genus $g>3$. Finding all Pfaffian representations of a given curve $C$ would provide a description of the open set $M_{C}\left(2, \mathcal{O}_{C}(d-\right.$

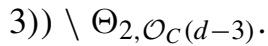

Vanhaecke's method could be used for smooth curves of higher genus. First we find all determinantal representations of $C$ (which are of smaller size than Pfaffians). Determinantal representations induce Pfaffian representations of the form

$$
\left[\begin{array}{cc}
0 & \diamond \\
-\diamond^{t} & 0
\end{array}\right]
$$

which by Section 5 correspond to decomposable bundles. These define the singular locus of the moduli space. The equations of the moduli space could be then found by integration.

It was asked by one of the referees (that found the paper not suitable to be published in another journal) if it was possible to use similar methods for a hyperelliptic curve of genus 3 to find explicit equations of the moduli space (the double quadric).

\section{References}

[1] E. Arbarello, M. Cornalba, P. Griffiths and J. Harris, "The Geometry of Algebraic Curves", Vol. 1, Grundlehren der mathematischen Wissenschaften, Vol. 267, Springer-Verlag, 1985.

[2] A. Beauville, Determinantal hypersurfaces, Michigan Math. J. 48 (2000), 39-63.

[3] A. BEAUVILle, Vector bundles on curves and generalized theta functions: recent results and open problems, In: "Current Topics in Complex Algebraic Geometry (Berkeley, CA, 1992/93)", Math. Sci. Res. Inst. Publ., Vol. 28, Cambridge Univ. Press, Cambridge, 1995, $17-33$.

[4] A. BUCKLEY, Elementary transformations of Pfaffian representations of plane curves, Linear Algebra Appl. 433 (2010), 758-780.

[5] R. J. COOK and A. D. ThomAs, Line bundles and homogeneous matrices, Quart. J. Math. Oxford Ser. (2) 30 (1979), 423-429.

[6] I. DolgACHEv, "Topics in Classical Algebraic Geometry", Lecture Notes http://www.math.lsa.umich.edu/ idolga/lecturenotes.html.

[7] J. M. Drezet and M. S. Narasimhan, Groupe de Picard des varietes de modules de fibres semi-stables sur les courbes algebriques, Invent. Math. 97 (1989), 53-94. 
[8] W. Fulton and P. Pragacz, "Shubert Varieties and Degeneracy Loci”, Lecture Notes in Mathematics, Vol. 1689, Springer-Verlag, 1998.

[9] R. Hartshorne, "Algebraic Geometry", Graduate Texts in Mathematics, Vol. 52, Springer-Verlag, 1977.

[10] M. MARCUS and R. WESTWICK, Linear maps on skew-symmetric matrices: The invariance of elementary symmetric functions, Pacific J. Math. (3) 14 (1960).

[11] P. LANCASTER and L. RODMAN, Canonical forms for symmetric / skew-symmetric real matrix pairs under strict equivalence and congruence, Linear Algebra Appl. 406 (2005), $1-76$.

[12] Y. LASZLO, À propos de l'espace des modules des fibrés de rang 2 sur une courbe, Math. Ann. 299 (1994), 597-608.

[13] R. LAZARSFELD, "Positivity in Algebraic Geometry II", A Series of Modern Surveys in Mathematics, Vol. 49, Springer-Verlag, 2000.

[14] M. S. NARASIMHAN and S. RAMANAN, $2 \theta$ linear systems on Abelian varieties, In: "Vector bundles on algebraic varieties (Bombay, 1984)", Stud. Math., Vol. 11, Tata Inst. Fund. Res., Bombay, 1987, 415-427.

[15] P. E. Newstead, "Introduction to Moduli Problems and Orbit Spaces", Tata Institute of Fundamental Research, Bombay, Springer-Verlag, 1978.

[16] W. M. Oxbury, C. Pauly and E. Previato, Subvarieties of $\mathcal{S U}_{C}(2)$ and $2 \theta$-divisors in the Jacobian, Trans. Amer. Math. Soc. 350 (1998), 3587-3614.

[17] J. Le Potier, "Lectures on Vector Bundles", Cambridge studies in advanced mathematics, Vol. 54, Cambridge University Press, 1997.

[18] C. S. SESHADRI, "Fibrés vectoriels sur les courbes algébriques", Astérisque, Vol. 96, 1982.

[19] I. R. Shafarevich, "Basic Algebraic Geometry 1", Springer-Verlag, 1994.

[20] M. THADDEUs, Conformal field theory and the cohomology of the moduli space of stable bundles, J. Differential Geom. 35 (1992), 131-149.

[21] P. VANHAECKE, Integrable systems and moduli spaces of rank two vector bundles on a non-hyperelliptic genus 3 curve, Ann. Inst. Fourier (Grenoble) 6 (2005), 1789-1802.

[22] V. VinNikov, Complete description of determinantal representations of smooth irreducible curves, Linear Algebra Appl. 125 (1989), 103-140.

[23] V. Vinnikov, "Determinantal Representations of Real Cubics and Canonical Forms of Corresponding Triples of Matrices", Mathematical Theory of Networks and Systems, Lecture Notes in Control and Inform. Sci., Vol. 58, 1984.

Department of Mathematics

Faculty of Mathematics and Physics

University of Ljubljana

Jadranska 19

1000 Ljubljana, Slovenia

anita.buckley@fmf.uni-lj.si

tomaz.kosir@fmf.uni-lj.si 